\title{
Cerebrospinal Fluid Galectin-1 Levels Discriminate Patients with Parkinsonism from Controls
}

\author{
Tainá M. Marques ${ }^{1,2,3} \cdot$ Anouke van Rumund ${ }^{1,3} \cdot$ Ilona B. Bruinsma ${ }^{1,2} \cdot$ Hans J. C. T. Wessels ${ }^{2} \cdot$ Jolein Gloerich $^{2}$. \\ Rianne A. J. Esselink ${ }^{1,3} \cdot$ Bastiaan R. Bloem ${ }^{1,3} \cdot$ H. Bea Kuiperij ${ }^{1,2} \cdot$ Marcel M. Verbeek $^{1,2,3}$ (D
}

Received: 22 May 2018 / Accepted: 13 November 2018 / Published online: 21 November 2018

(C) The Author(s) 2018

\begin{abstract}
Parkinson's disease (PD) is the second most common neurodegenerative disorder in elderly people. Currently, the diagnosis of PD is based on neurological examination, neuroimaging, and the response to dopaminergic medication. The diagnosis can be challenging, especially at early disease stages, when the symptoms of patients with atypical parkinsonism (APD) may strongly overlap. Therefore, reliable biomarkers that are able to identify patients with PD are much needed. Here, we aimed to identify and validate new biomarkers for PD in cerebrospinal fluid (CSF). We performed a profiling experiment using mass spectrometry (MS) of CSF from ten PD patients and ten matched non-neurological controls. We selected one protein, galectin-1 (Gal-1), which was differentially expressed in PD vs. controls, and quantified its concentrations in CSF by enzyme-linked immunosorbent assay (ELISA) in three new cohorts of 37 PD patients, 21 APD patients, and 44 controls. CSF levels of Gal-1 were lower in PD in both the discovery and validation experiments and discriminated PD from controls with moderate-high accuracy levels (ELISA: area under the curve $=0.7$ ). Similar levels of Gal-1 were found in PD and APD. Gal-1 levels were correlated to age in all groups and correlated in the PD patients to CSF levels of total tau, phosphorylated tau, neurofilament light chain (NFL), and the mini-mental state examination (MMSE) score. We conclude that MS profiling of proteins may be a useful tool to identify novel biomarkers of neurological diseases and that CSF Gal-1 levels may discriminate PD from non-neurological controls.
\end{abstract}

Keywords Parkinson's disease $\cdot$ Biomarkers $\cdot$ Galectin- $1 \cdot$ Cerebrospinal fluid $\cdot$ Validation

\section{Introduction}

Parkinson's disease (PD) is one of the $\alpha$-synucleinopathies and the most prevalent neurodegenerative movement disorder. Accumulation of $\alpha$-synuclein ( $\alpha$-syn) in dopaminergic neurons in PD leads to neuronal death causing motor and non-motor dysfunction. PD motor symptoms include bradykinesia,

Electronic supplementary material The online version of this article (https://doi.org/10.1007/s12035-018-1426-9) contains supplementary material, which is available to authorized users.

Marcel M. Verbeek

Marcel.Verbeek@radboudumc.nl; http://www.neurochemistry.nl

1 Department of Neurology, Donders Institute for Brain, Cognition and Behaviour, Radboud University Medical Center, Nijmegen, The Netherlands

2 Department of Laboratory Medicine, Radboud University Medical Center, Nijmegen, The Netherlands

3 Parkinson Center Nijmegen, Nijmegen, The Netherlands muscular rigidity, impaired balance, and resting tremor. Nonmotor symptoms include sleep disorders, olfactory dysfunction, autonomic dysfunction, and cognitive impairment $[1,2]$.

PD diagnosis is based on neurological evaluations, neuroimaging, and the response to dopaminergic medication following the current international clinical criteria [3, 4]. However, especially at early stages of diseases, the symptoms and signs of patients with atypical parkinsonisms (APD), such as multiple system atrophy (MSA) and progressive supranuclear palsy (PSP), may strongly overlap with PD, leading to misdiagnosis and incorrect choice of treatment. Therefore, reliable biomarkers that could identify PD are dearly needed.

Cerebrospinal fluid (CSF) is the body fluid that is closest to the brain, and its composition may reflect the (pathological) processes in the brain. Several proteins that were already known to be involved in PD have been investigated in CSF as potential biomarkers for diagnosis, disease progression, or cognitive decline, such as $\alpha$-syn, neurofilament light chain (NFL), DJ-1, tau, and amyloid $\beta 42$ (reviewed in [5]). A consistent moderate reduction in CSF $\alpha$-syn levels has been described not only in PD 
but also in other $\alpha$-synucleinopathies (reviewed in [6]). Although many studies have been identified potential biomarkers for PD, none of them has yet reached clinical practice.

We aimed to identify new CSF biomarkers that have the potential to discriminate PD from controls. For this purpose, we selected PD patients from a unique cohort of patients with uncertain diagnosis of parkinsonism at presentation, which is very representative of the daily situations when clinicians are confronted with patients with the suspicion of a movement disorder at the first visit to a specialist. We performed a profiling experiment by mass spectrometry in CSF of ten PD patients and ten non-neurological controls. We selected the protein galectin-1 (Gal-1), which was differentially expressed in PD vs. controls, for further validation studies, using an enzyme-linked immunosorbent assay (ELISA).

\section{Methods}

\section{Patients and CSF}

We have selected CSF samples from ten PD and ten nonneurological control patients for the discovery experiment matched for sex and age. For the validation experiment, we have included CSF from 37 PD patients, 21 APD patients (MSA $=14$; PSP $=7$ ), and 44 non-neurological controls. Samples were matched for sex in all groups and for age in PD and controls, since age was higher in the APD group. We used separate cohorts of patients for the discovery and validation phases of the study, with the exception of only one PD CSF sample, which was used for both discovery and validation experiments. CSF samples for the discovery experiment were also selected based on low number of leukocytes (0-5 cells per $\mu \mathrm{L})$ and erythrocytes $(\leq 200$ cells per $\mu \mathrm{L})$. Demographic characteristics for both cohorts are shown in Table 1.

PD and APD patients for the discovery and validation cohorts were selected from a longitudinal study performed at the Radboud University Medical Center, previously described in detail (Nijmegen, The Netherlands) [7]. These cohorts included patients who were referred to our tertiary center between January 2003 and December 2006 with an uncertain and yet undefined diagnosis of parkinsonism. At baseline, an extensive array of ancillary diagnostic tests was performed, among which a lumbar puncture to allow biomarker studies. These patients had been followed up for 3 years at each time a team of movement disorders specialists determined a final clinical diagnosis. The diagnosis of PD or APD was based on established criteria for PD [8], MSA [9], or PSP [10] at the time of 3-year follow-up and updated according to the most recent clinical criteria $[3,4,11]$. Clinical parameters were obtained from PD and APD patients both at baseline and after 3 years of follow-up, including disease severity and cognitive function, using the Hoehn and Yahr (H\&Y) scores [12], Unified Parkinson's Disease Rating Scale (UPDRS) [13], International Cooperative Ataxia Rating Scale (ICARS) [14], and mini-mental state examination (MMSE) [15]. The use of dopaminergic medication was registered at the time of lumbar puncture, in order to include as a possible confounding factor. CSF levels of $\alpha$-syn, total tau, phosphorylated tau, and NFL concentrations in the PD patients were previously published by our group by using various ELISAs (see Table 1) [16-18]. Details of the methods for the quantification of these CSF parameters have been described in detail [16-18].

The non-neurological control group consisted of patients who were referred to our center with a suspicion of neurological disease, but after extensive neurological examination had no neurological disorder and were diagnosed with other disorders, such as non-neuronal sarcoidosis, diabetes, radiculopathy, or headache. Their CSF did not show any abnormality for the following parameters: cell count, glucose, total protein, lactate, hemoglobin, bilirubin, and oligoclonal IgG bands.

CSF samples of PD, APD, and non-neurological controls were collected in polypropylene tubes, centrifuged, aliquoted, and stored in polypropylene tubes at $-80{ }^{\circ} \mathrm{C}$ until experiments. All participants provided written informed consent, and the study was approved by the local institutional review board Arnhem-Nijmegen.

\section{Mass Spectrometry Profiling}

Total protein concentration in CSF was determined using the 2D Quant kit (GE Healthcare Life Sciences, UK), according to the manufacture's protocol, and $400 \mu \mathrm{g}$ of total protein for each sample was used as input for the discovery experiment. CSF samples were applied to an affinity removal column for depletion of 14 most abundant proteins (MARS-14, Agilent Technologies, Santa Clara, CA, USA) to enrich low-abundant proteins.

Samples were diluted in $8 \mathrm{M}$ urea to denature proteins prior to reduction in $10 \mathrm{mM}$ dithiotreitol for $20 \mathrm{~min}$ at room temperature and alkylation with $50 \mathrm{mM}$ chloroacetamide for $20 \mathrm{~min}$ at room temperature in the dark. Proteolytical digestion was performed by a first incubation with LysC protease for $3 \mathrm{~h}$ at $37^{\circ} \mathrm{C}$ after which the sample was diluted 1:3 with $50 \mathrm{mM}$ ammonium bicarbonate prior to overnight incubation with trypsin at $37{ }^{\circ} \mathrm{C}$. Peptides were concentrated and desalted using C18 Omix tips (Agilent Technologies), eluted in $20 \mu \mathrm{L} 80 \%$ acetonitrile, and dried using a SpeedVac centrifuge at $45^{\circ} \mathrm{C}$. Peptides were suspended in ammonium hydroxide buffer ( $\mathrm{pH} 10)$ and subsequently fractionated using C18-reversed phase liquid chromatography (Waters Acquity UPLC; Waters Xbridge C18 3.5- $\mu \mathrm{m}$ particles, $1.0-\mathrm{mm} \mathrm{ID} \times 100-\mathrm{mm}$ length). Peptides were eluted from the column at $100 \mu \mathrm{L} / \mathrm{min}$ using a 15 -min linear gradient of 5 to $45 \%$ acetonitrile adjusted to $\mathrm{pH} 10$ with ammonium hydroxide. 
Table 1 Group characteristics

Discovery

Control

Demographics

n

Age at inclusion (years)

Sex (male/female)

Disease duration (months)

DM (no/yes)

CSF parameters

Gal-1 $^{\#}$

Total protein $(\mathrm{mg} / \mathrm{L})$

Gal-1/total protein (ng/mg)

$\alpha$-Syn $(\mu \mathrm{g} / \mathrm{L})$

NFL (ng/L)

Total tau (ng/L)

Phosphorylated tau (ng/L)

Disease severity

Baseline

H\&Y score

UPDRS score

ICARS score

MMSE score

Follow-up

$\mathrm{H} \& \mathrm{Y}$ score

UPDRS score

ICARS score

MMSE score

NA $\quad 58 \pm 27$

10
$59 \pm 7$
$6 / 4$
NA
NA

$5 \mathrm{E}+06 \pm 4 \mathrm{E}+06$

$468 \pm 66$

NA

NA

NA

NA

$1415 \pm 528$

$58 \pm 27$

NA

NA

$n=10$

$2 \pm 0$

$n=10$

$22 \pm 6$

NA

$n=9$

$2 \pm 2$

NA

$n=10$

$29 \pm 1$

NA

$n=9$

$2 \pm 1$

NA

$n=7$

$28 \pm 10$

NA

$n=7$

$2 \pm 1$

NA

$n=8$
Validation

Control PD APD $\quad p$ value*

$44 \quad 37 \quad 21$

$58 \pm 10 \quad 57 \pm 10$

$p=0.4$

$58 \pm 10$

$57 \pm 10$

$24 / 13$

NA

NA

$36 \pm 34$

$31 / 6$

$64 \pm 7$

$13 / 8$

$31 \pm 23$

$14 / 7$

$\begin{array}{ll}1 & \\ 3 / 8 & p=0.02 \\ 1 \pm 23 & p=0.26 \\ 4 / 7 & p=0.92\end{array}$

$6961 \pm 3475$

$6046 \pm 2631$

$7133 \pm 3795$

$530 \pm 182$

$547 \pm 644$

$11 \pm 5$

$591 \pm 273$

$p=0.46$

$17 \pm 9$

$n=37$

$29 \pm 12$

$n=37$

$1122 \pm 639$

$14 \pm 9$

$p<0.001$

NA

$n=20$

$30 \pm 12$

$n=20$

$4511 \pm 3633$

$n=37$

$202 \pm 71$

$n=21$

$260 \pm 111$

$n=37$

$48 \pm 16$

$n=21$

$49 \pm 15$

$p=0.003$

$p=0.60$

$p<0.0001$

$p=0.55$

$p=0.92$

NA

$n=36$

$2 \pm 1$

NA

$n=35$

$26 \pm 13$

$3 \pm 1$

$n=21$

$33 \pm 13$

NA

$n=33$

$2 \pm 3$

$n=16$

$10 \pm 11$

$n=37$

$28 \pm 2$

$n=20$

$27 \pm 3$

$p<0.0001$

$p=0.046$

$p<0.001$

NA

NA

$n=35$

$2 \pm 1$

$n=14$

$4 \pm 1$

$n=11$

$n=33$

$37 \pm 9$

$29 \pm 13$

$n=11$

$12 \pm 10$

$3 \pm 3$

$29 \pm 1$

$n=11$

$26 \pm 3$

$p=0.10$

$p<0.0001$

$p=0.049$

$p<0.001$

NA

$n=30$

$28 \pm 3$

Values are expressed as mean \pm standard deviation

$n$ number of samples, $D M$ intake of dopaminergic medication at CSF collection, CSF cerebrospinal fluid, Gal-1 galectin-1, $\alpha$-syn $\alpha$-synuclein, $P D$ Parkinson's disease, APD atypical parkinsonism, NA not applicable, H\&Y Hoehn and Yahr score, UPDRS Unified Parkinson's Disease Rating Scale, ICARS International Cooperative Ataxia Rating Scale, MMSE mini-mental state examination score

${ }^{\#}$ Units: in discovery cohorts, arbitrary intensity; in validation cohorts, $\mathrm{ng} / \mathrm{L}$

*Parameters were analyzed with Kruskal-Wallis test when data of the three groups was available. For comparisons between two groups Student's $t$ test or Mann-Whitney $U$ test, except for sex, which was analyzed using chi-squared test; In italic $p$-value below 0.05

The 20 collected fractions were adjusted to $\mathrm{pH} 2.7$ using formic acid prior to LC-MS/MS analysis.

Samples were analyzed by nanoflow liquid chromatography (Bruker Daltonics; nano-Advance) connected online to an ultra high-resolution quadrupole time-of-flight tandem mass spectrometer (Bruker Daltonics; maXis 4G ETD) via an axial desolvation vacuum-assisted electrospray ionization source (Bruker Daltonics; Captive Sprayer). Peptides were loaded onto the trapping column (Acclaim PepMap 100, $75 \mu \mathrm{m} \times$ $2 \mathrm{~cm}$, nanoViper, $3-\mu \mathrm{m}$ 100- $\AA$ C18 particles; Thermo 
Scientific) at $10 \mu \mathrm{L} / \mathrm{min}$ with $0.1 \%$ formic acid using two loop volumes of solvent $(20 \mu \mathrm{L})$. Peptides were eluted from the analytical column (Acclaim PepMap RSLC, $75 \mu \mathrm{m} \times 15 \mathrm{~cm}$, nanoViper, $2-\mu \mathrm{m} 100-\AA$ C18 particles; Thermo scientific) using a 20 -min linear gradient of 5 to $35 \%$ acetonitrile in $0.1 \%$ formic acid at $600 \mathrm{~nL} / \mathrm{min}$. The mass spectrometer was operated in positive ion mode for data-dependent MS/MS acquisition. Each AutoMSn duty cycle consisted of one full MS spectrum (150-3700 m/z, 2-Hz spectrum acquisition rate) followed by six data-dependent MS/MS experiments acquired at intensity scaled spectral acquisition rates $(3 \mathrm{~Hz}$ at 2000 counts, $16 \mathrm{~Hz}$ at 100,000 counts). Only precursor ions in the range of $400-1400 \mathrm{~m} / \mathrm{z}$ with charge state of $z=2+$ or higher were considered for collision-induced dissociation experiments with dynamic exclusion set to $2 \mathrm{~min}$.

Raw MS data files were subsequently analyzed by MaxQuant software version 1.5 [19] using the predefined Qq-ToF parameter settings against the RefSeq (release 55) human protein sequence database with added contaminant protein sequences. Cysteine carbamidomethylation was specified as fixed modification whereas protein $\mathrm{N}$-terminal acetylation, methionine oxidation, and deamidation of glutamine and/or asparagine as variable modifications. Label-free quantitation was performed using match between runs and requantify options using at least two razor and unique peptides. False discovery rate tolerances were set to 0.01 at both the peptide and protein level. Putative protein biomarkers were considered for subsequent validation based on $p$ values calculated using the Mann-Whitney $U$ test $(p<0.05)$ and should be quantified in at least $50 \%$ of the samples of any group.

\section{Gal-1 ELISA}

Gal-1 was quantified in CSF by using a commercial ELISA (Human Galectin-1 PicoKine ${ }^{\text {TM }}$ ELISA Kit; Boster Biological Technology, Pleasanton, CA, USA, Catalog \#EK0762), according to the company's protocol, with the exception of prolonged incubation times, as suggested by the company. Biotinylated anti-human galectin-1 antibody was incubated for $90 \mathrm{~min}$, avidin-biotin-peroxidase complex (ABC) for $50 \mathrm{~min}$, and color-developing agent for $30 \mathrm{~min}$. CSF was diluted three times, and all samples were analyzed in duplicate.

We performed a partial validation of the ELISA according to previous recommendations $[20,21]$. The detection limit was determined by measuring 18 blanks within one plate followed by calculation of the concentration that corresponds to the mean of all blanks plus three times the standard deviation.

The recovery was evaluated by spiking two different concentrations $(6.7 \mathrm{ng} / \mathrm{mL}$ and $4.5 \mathrm{ng} / \mathrm{mL})$ of Gal- 1 recombinant protein in three times diluted CSF samples or in sample buffer. The percentage of spiked recombinant protein that was recovered from CSF was calculated. Values between $80 \%$ and $120 \%$ were considered satisfactory.

Precision was determined based on (1) intra-assay variation by measuring five diluted CSF samples in four replicates within one plate, and (2) inter-assay variation by measuring three diluted CSF samples in duplicate at identical positions in five different plates on five different days. The mean coefficient of variation $(\mathrm{CV})$ was calculated; a CV below $20 \%$ was considered satisfactory.

CSF Gal-1 concentrations in the validation cohort were normalized by CSF total protein concentration to correct for a small significant difference of total protein concentration between PD (mean $530 \pm 182 \mathrm{mg} / \mathrm{L}$ ), APD (mean $591 \pm$ $273 \mathrm{mg} / \mathrm{L}$ ), and controls (mean $547 \pm 652 \mathrm{mg} / \mathrm{L}, p=0.004$ ). Total protein concentration in CSF was measured by turbidimetric benzethonium chloride method using a Cobas 8000 instrument (Roche Diagnostics, Switzerland) for automated measurement.

\section{Data Analysis}

Data analysis was performed using IBM SPSS Statistics 22 (Armonk, NY, USA) and GraphPad Prism 5 (La Jolla, CA, USA). Differences between groups were determined by ANOVA followed by Bonferroni post hoc test for normally distributed data and Kruskal-Wallis test followed by Dunn's post hoc test for not normally distributed data. Mann-Whitney $U$ test (in case of non-parametric data distribution) or Student's $t$ test (for parametric data) was used when data of only two groups were available. Analysis of covariance was performed with age and intake of dopaminergic medication as confounding factors. Correlations and partial correlations, with age and total protein concentration as covariates, were investigated by Spearman's test. Receiver operating characteristic (ROC) curve was constructed to determine the diagnostic accuracy by the area under the curve (AUC) and the Youden index for the optimal cutoff values of sensitivity and specificity.

\section{Results}

The aim of our study was to identify and validate a new biomarker for PD diagnosis. We first performed a profiling experiment by mass spectrometry in CSF of ten PD and ten nonneurological control patients. This discovery experiment resulted in the identification of 5543 peptides in CSF of both groups, corresponding to 872 different identified proteins. Only proteins quantified in at least five out of ten patients in any group (PD or control) were considered for further analysis, resulting in 482 proteins to be analyzed. Among these, 32 proteins were present at significantly different levels in PD and controls $(p<0.05)$ (Table S1). More details about the 
identification of peptides and proteins will be described in a separate study (manuscript in preparation). We selected Gal-1, which was present at significantly lower concentrations in PD ( $8 \times$-fold lower), for further validation as a CSF biomarker candidate for the discrimination of PD and controls $(p=$ 0.02) (Fig. 1a).

The performance of the ELISA assay was as follows. The detection limit was determined at $460 \mathrm{ng} / \mathrm{L}$. The recovery of recombinant Gal-1 protein spiked in CSF was considered satisfactory with $\mathrm{CV}$ ranging from 85 to $118 \%$. Both the intraassay and inter-assay variation were considered satisfactory with a mean $\mathrm{CV} \%$ of $4 \%( \pm 0.01)$ and $14 \%( \pm 0.05)$, respectively.

Age was significantly different between groups due to older patients in the APD group $(p=0.02)$. Total protein concentration was lower in PD and higher in APD patients than in controls $(p<0.001)$; therefore, Gal-1 levels were corrected for the total protein level. Gal-1 levels were lower in PD (mean $11 \mathrm{ng} / \mathrm{mg}$ total protein) compared to non-neurological controls

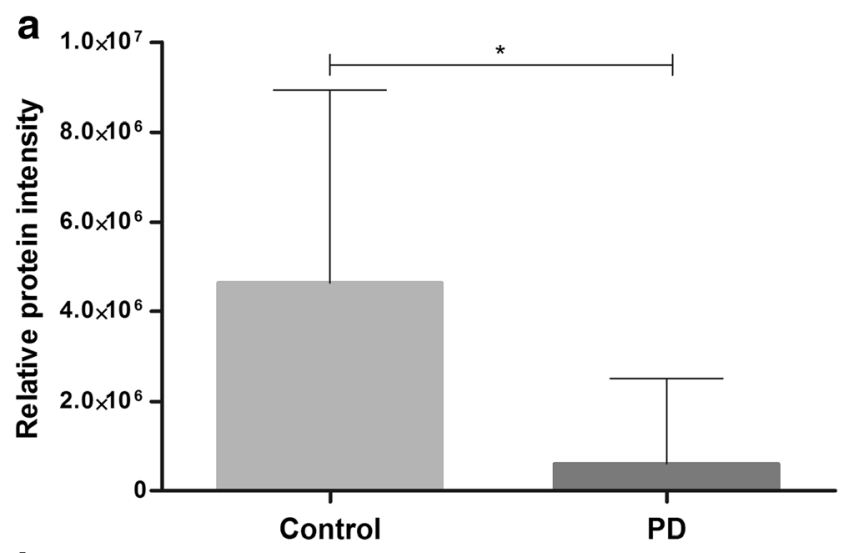

b

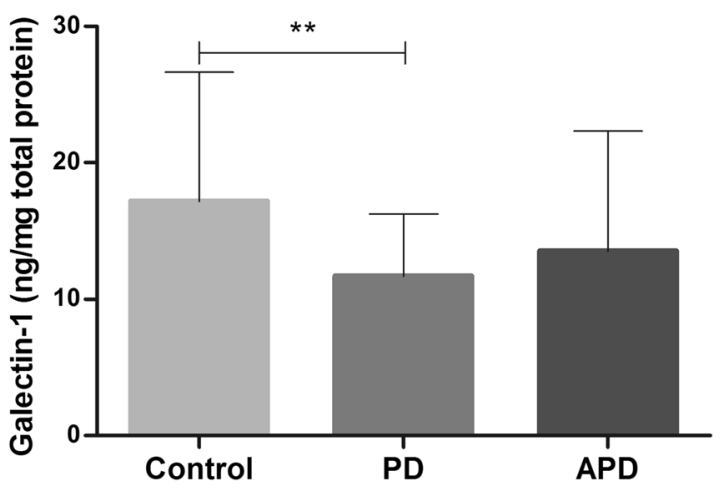

Fig. 1 CSF Gal-1 levels in discovery (a) and validation (b) experiments for PD, APD, and non-neurological controls. a Relative protein intensity of Gal-1 found in profiling experiment by mass spectrometry showed decreased Gal-1 levels in PD compared to controls. b Gal-1 levels in CSF are lower in PD compared to controls in the validation study, but similar with APD. Gal-1 CSF levels were quantified by ELISA and normalized by total protein concentration. Data were analyzed using MannWhitney $U$ test for discovery and Kruskal-Wallis test for validation; mean levels are shown with standard deviation, $* p<0.05 ; * * p<0.001$ (mean $17 \mathrm{ng} / \mathrm{mg}$ total protein, $p<0.003$; Fig. 1b), but similar to APD (mean $14 \mathrm{ng} / \mathrm{mg}$ total protein). Gal-1 levels were similar in men and women in each group. Of note, Gal-1 levels in PD, APD, and controls were correlated with age (rho $=0.44, p<0.0001$ ). The difference in Gal-1 levels between the PD and controls remained significant after correction for age and intake of dopaminergic medication ( $p=$ 0.013). CSF Gal-1 levels in PD/APD patients were similar when they were either on dopaminergic medication or not at the time of lumbar puncture $(p=0.15)$. CSF Gal-1 levels in the PD group were positively correlated to CSF concentrations of total tau (rho $=0.53, p<0.001)$, phosphorylated tau (rho $=0.54, p<0.001$ ), and NFL (rho $=0.58, p<0.0001$ ), but $\alpha$-syn levels were not significantly correlated to Gal-1 (rho = $-0.11, p=0.5)$. CSF Gal-1 levels correlated to MMSE scores determined at 3 years of follow-up (rho $=-0.44, p=0.02$; Fig. 2). Gal-1 CSF levels in the APD group were correlated only to disease duration ( $r h o=0.45, p=0.04$ ). No significant correlations between Gal-1 and other clinical parameters were observed. Partial analysis, taking age and total protein concentration as covariates, confirmed the correlations of Gal-1 in the PD group with total tau, phosphorylated tau, but not with NFL or MMSE. Correlation of Gal-1 levels to disease duration in the APD group was also retained.

\section{Discussion}

We aimed to identify new CSF biomarkers for PD. We successfully performed a mass spectrometry profiling study using CSF of PD and controls, in which a total of 482 proteins were robustly identified. Among them, Gal-1 was considered as a potential biomarker for PD due to the strongly decreased levels in PD as compared to controls. We confirmed these differences by ELISA in an independent and larger validation cohort of PD, APD, and controls. Gal-1 levels in CSF had a moderate-high accuracy for discrimination of PD and controls. CSF Gal-1 levels were similar in PD and APD, however, indicating that Gal-1 levels in CSF may serve as a biomarker for parkinsonism, rather than for PD only.

Gal-1 is a member of the galectin family of proteins that binds to $\beta$-galactosides sugars. It is expressed in the nervous system during development, and a few other studies have reported expression of Gal-1 in the central and peripheral nervous system in adults [22-24]. Gal-1 expression in the nervous system has been correlated to proliferation of adult neural stem cells, astrocyte differentiation, and inflammation $[25,26]$.

In several reports, a neuroprotective role for Gal-1 has been described. Expression of Gal-1 was previously related to axonal and nerve cell regeneration after injury in animal models $[23,27,28]$. Administration of Gal-1 in an animal model of amyotrophic lateral sclerosis was associated with regeneration 

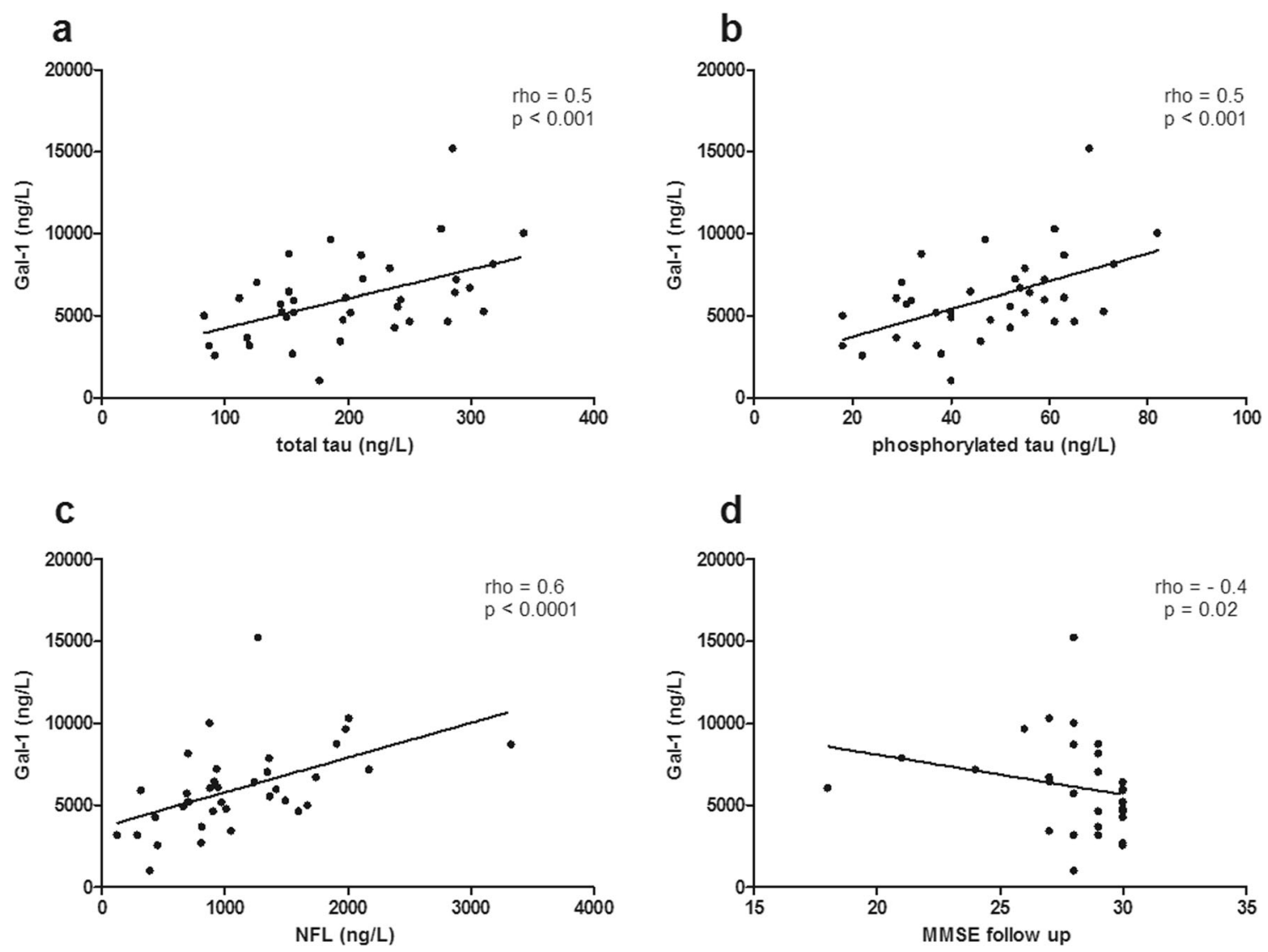

Fig. 2 Correlation analysis in the PD group between Gal-1 and total tau, phosphorylated tau, NFL, and MMSE score. PD CSF Gal-1 levels were positively correlated to total tau (a), phosphorylated tau (b), and NFL (c).

of spinal motor neurons, improvement of motor symptoms, and delay of disease onset [29].

The role of Gal-1 in PD or in APD is not yet clear, and only two studies reported findings in PD. One study showed reduction of Gal-1 levels in a cell model of PD, consisting of PC12 cells that were treated with a proteasomal inhibitor to promote ubiquitin-proteasome dysfunction, reproducing a general characteristic of PD [30]. Reduction of Gal-1 in this PD cell model may indicate the loss of neuroprotection after proteasomal inhibition. In another study, high Gal-1 levels were reported in PD substantia nigra [31]. These results are in apparent contrast with our study, since we observed decreased Gal-1 concentrations in the CSF of PD patients. The tissue study comprised five PD and five controls patients, with age ranging from 73 to 92 years in the PD group. No information about disease duration or other clinical parameters could be retrieved from this study, but at high age, PD is typically advanced to a severe stage. Thus, the contradictory outcomes of Gal-1 protein levels in brain tissue vs. CSF may be explained by the difference in sample type, a difference in disease stage (relatively early in our study vs. relatively late in the tissue study), and the younger population in our study which may affect Gal-1 concentrations in CSF. No reports

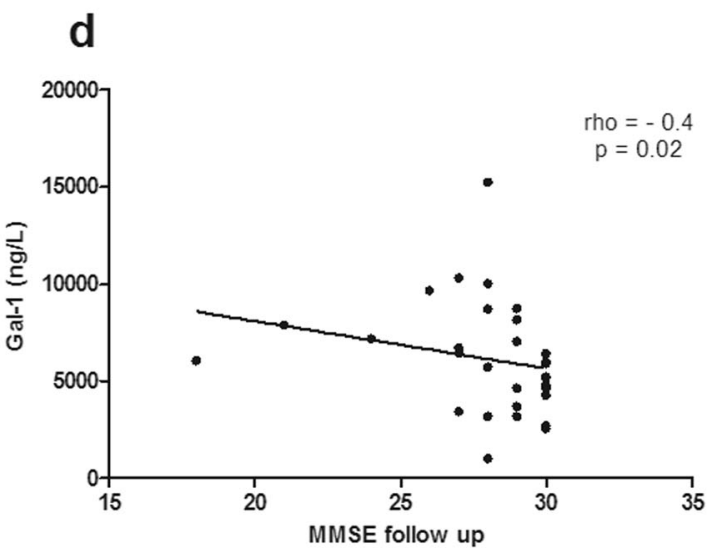

A negative correlation was found between Gal-1 and MMSE, a clinical parameter for cognitive impairment (d). Spearman's rho coefficient value and $p$ value for each correlation are indicated in the graphs

have been published about a relation between Gal-1 protein levels in different brain regions as compared to CSF, or a relation between age and tissue levels of Gal-1.

The correlation of Gal-1 with either tau or NFL may indicate association with neuronal degeneration. Tau has previously been described as a potential biomarker for prediction of cognitive decline in PD, but this was not a consistent finding across multiple studies (reviewed in [32]). Overexpression of Gal-1 in mice with spinal lesions was correlated to an increase of tau levels and axonal regeneration of injured axons [33]. NFL has been widely studied as a biomarker for discrimination of PD from APDs with high accuracy levels (reviewed in [32]). Previous studies also indicated the involvement of NFL in axonal regeneration (reviewed in [34]). Based on literature, the correlation of Gal-1 with either tau or NFL, but not with $\alpha$-syn levels in CSF, allows us to speculate a potential association of Gal-1 with axonal damage. However, future studies should be performed to investigate in more detail such as possible relation.

One limitation to our study may be the relatively small number of APD samples available for this study, which might have underestimated the power of Gal-1 to discriminate PD 
from APD. However, the design of our prospective study did not allow for an inclusion of a larger number of APD patients. Further confirmation in larger, independent, cohorts remains necessary.

Another limitation is that our CSF samples were stored for a long period since its collection, between 2003 and 2006, and long-term storage may affect protein stability. Protein levels are generally low in CSF, and differences in sample processing could affect the results of mass spectrometry and ELISA analysis. However, we followed international guidelines for CSF collection and storage that were described later in a consensus paper [35]. Previous studies reported increased protein instability in CSF samples storage at $-20{ }^{\circ} \mathrm{C}$ [35]; however, our samples were stored at $-80^{\circ} \mathrm{C}$. A recent study with Alzheimer's disease CSF samples stored at $-80^{\circ} \mathrm{C}$ for up to 12 years showed that CSF concentration of amyloid $\beta$, total, and phosphorylated tau proteins remained stable during this CSF storage time [36]. Therefore, we assume that the extended storage time has not influenced our results.

An important strong aspect of our study is that, although the number of parkinsonism patients we included in this study is moderate, they were selected from a unique longitudinal study. Unlike many other biomarker studies, which use highly selected cohorts of patients with clinically undisputable diagnosis, this study included patients with uncertain diagnosis at baseline, closely representing the daily situation for clinicians when they are confronted with a patient suspected of a movement disorder [7]. In these situations, biomarkers are mostly needed. Therefore, our cohort offers a great and relevant basis for biomarker discovery and validation.

In summary, we successfully profiled proteins present in CSF of PD and non-neurological controls by mass spectrometry. Among the proteins that were differentially expressed in PD vs. controls, Gal-1 was selected as a potential biomarker for PD. Our validation experiment for Gal-1 confirmed our findings from the discovery study, indicating that mass spectrometry profiling of proteins in CSF may be a useful tool to indentify novel biomarkers of neurological diseases, but this validation also demonstrated that CSF Gal-1 levels were similar in PD and APD. Furthermore, the correlations of Gal-1 with both NFL and tau suggested that Gal-1 may be involved in axonal function; however, further studies should clarify this association.

Acknowledgements We thank Iris Kersten, Alexandra Versleijen, Laura van Meurs, and Maruelle Luimes for helping on the development of this project.

Funding Information Tainá Macherini Marques is supported by Conselho Nacional de Desenvolvimento Científico e Tecnológico (CNPq), Brazil [grant no. 249032/2013-9].
Compliance with Ethical StandardsAll participants provided written informed consent, and the study was approved by the local institutional review board Arnhem-Nijmegen.

Conflict of Interest The authors declare that they have no conflict of interest.

Open Access This article is distributed under the terms of the Creative Commons Attribution 4.0 International License (http:// creativecommons.org/licenses/by/4.0/), which permits unrestricted use, distribution, and reproduction in any medium, provided you give appropriate credit to the original author(s) and the source, provide a link to the Creative Commons license, and indicate if changes were made.

\section{References}

1. Kalia LV, Lang AE (2015) Parkinson's disease. Lancet 386(9996): 896-912. https://doi.org/10.1016/S0140-6736(14)61393-3

2. Poewe W (2008) Non-motor symptoms in Parkinson's disease. Eur J Neurol 15(Suppl 1):14-20. https://doi.org/10.1111/j.1468-1331. 2008.02056.x

3. Postuma RB, Berg D, Adler CH, Bloem BR, Chan P, Deuschl G, Gasser T, Goetz CG et al (2016) The new definition and diagnostic criteria of Parkinson's disease. Lancet Neurol 15(6):546-548. https://doi.org/10.1016/s1474-4422(16)00116-2

4. Postuma RB, Berg D, Stern M, Poewe W, Olanow CW, Oertel W, Obeso J, Marek K et al (2015) MDS clinical diagnostic criteria for Parkinson's disease. Mov Disord 30(12):1591-1601. https://doi. org/10.1002/mds.26424

5. Magdalinou N, Lees AJ, Zetterberg H (2014) Cerebrospinal fluid biomarkers in parkinsonian conditions: an update and future directions. J Neurol Neurosurg Psychiatry 85(10):1065-1075. https:// doi.org/10.1136/jnnp-2013-307539

6. Simonsen AH, Kuiperij B, El-Agnaf OM, Engelborghs S, Herukka SK, Parnetti L, Rektorova I, Vanmechelen E et al (2016) The utility of alpha-synuclein as biofluid marker in neurodegenerative diseases: a systematic review of the literature. Biomark Med 10(1): 19-34. https://doi.org/10.2217/bmm.14.105

7. Aerts MB, Esselink RA, Abdo WF, Meijer FJ, Drost G, Norgren N, Janssen MJ, Borm GF et al (2015) Ancillary investigations to diagnose parkinsonism: a prospective clinical study. J Neurol 262(2): 346-356. https://doi.org/10.1007/s00415-014-7568-4

8. Hughes AJ, Daniel SE, Kilford L, Lees AJ (1992) Accuracy of clinical diagnosis of idiopathic Parkinson's disease: a clinicopathological study of 100 cases. J Neurol Neurosurg Psychiatry 55(3):181-184

9. Gilman S, Wenning GK, Low PA, Brooks DJ, Mathias CJ, Trojanowski JQ, Wood NW, Colosimo C et al (2008) Second consensus statement on the diagnosis of multiple system atrophy. Neurology 71(9):670-676. https://doi.org/10.1212/01.wnl. 0000324625.00404 .15

10. Litvan I, Agid Y, Calne D, Campbell G, Dubois B, Duvoisin RC, Goetz CG, Golbe LI et al (1996) Clinical research criteria for the diagnosis of progressive supranuclear palsy (Steele-RichardsonOlszewski syndrome): report of the NINDS-SPSP international workshop. Neurology 47(1):1-9

11. Hoglinger GU, Respondek G, Stamelou M, Kurz C, Josephs KA, Lang AE, Mollenhauer B, Muller U, Nilsson C, Whitwell JL, Arzberger T, Englund E, Gelpi E, Giese A, Irwin DJ, Meissner WG, Pantelyat A, Rajput A, van Swieten JC, Troakes C, Antonini A, Bhatia KP, Bordelon Y, Compta Y, Corvol JC, Colosimo C, Dickson DW, Dodel R, Ferguson L, Grossman M, Kassubek J, Krismer F, Levin J, Lorenzl S, Morris HR, Nestor P, Oertel WH, 
Poewe W, Rabinovici G, Rowe JB, Schellenberg GD, Seppi K, van Eimeren T, Wenning GK, Boxer AL, Golbe LI, Litvan I, Movement Disorder Society-endorsed PSPSG (2017) Clinical diagnosis of progressive supranuclear palsy: The movement disorder society criteria. Mov Disord 32 (6):853-864. doi:https://doi.org/10.1002/ mds. 26987

12. Hoehn MM, Yahr MD (2001) Parkinsonism: onset, progression, and mortality. 1967. Neurology 57(10 Suppl 3):S11-S26

13. Movement Disorder Society Task Force on Rating Scales for Parkinson's D (2003) The Unified Parkinson's Disease Rating Scale (UPDRS): status and recommendations. Mov Disord 18(7): 738-750. https://doi.org/10.1002/mds.10473

14. Trouillas P, Takayanagi T, Hallett M, Currier RD, Subramony SH, Wessel K, Bryer A, Diener HC et al (1997) International Cooperative Ataxia Rating Scale for pharmacological assessment of the cerebellar syndrome. The Ataxia Neuropharmacology Committee of the World Federation of Neurology. J Neurol Sci 145(2):205-211

15. Folstein MF, Robins LN, Helzer JE (1983) The mini-mental state examination. Arch Gen Psychiatry 40(7):812

16. Herbert MK, Eeftens JM, Aerts MB, Esselink RA, Bloem BR, Kuiperij HB, Verbeek MM (2014) CSF levels of DJ-1 and tau distinguish MSA patients from PD patients and controls. Parkinsonism Relat Disord 20(1):112-115. https://doi.org/10. 1016/j.parkreldis.2013.09.003

17. Herbert MK, Aerts MB, Beenes M, Norgren N, Esselink RA, Bloem BR, Kuiperij HB, Verbeek MM (2015) CSF neurofilament light chain but not FLT3 ligand discriminates parkinsonian disorders. Front Neurol 6:91. https://doi.org/10.3389/fneur.2015.00091

18. Aerts MB, Esselink RA, Abdo WF, Bloem BR, Verbeek MM (2012) CSF alpha-synuclein does not differentiate between parkinsonian disorders. Neurobiol Aging 33(2):430-e431-433. https:// doi.org/10.1016/j.neurobiolaging.2010.12.001

19. Cox J, Mann M (2008) MaxQuant enables high peptide identification rates, individualized p.p.b.-range mass accuracies and proteome-wide protein quantification. Nat Biotechnol 26(12): 1367-1372. https://doi.org/10.1038/nbt.1511

20. Andreasson U, Perret-Liaudet A, van Waalwijk van Doorn LJ, Blennow K, Chiasserini D, Engelborghs S, Fladby T, Genc S et al (2015) A practical guide to immunoassay method validation. Front Neurol 6:179. https://doi.org/10.3389/fneur.2015.00179

21. van Waalwijk van Doorn LJ, Koel-Simmelink MJ, Haussmann U, Klafki H, Struyfs H, Linning P, Knolker HJ, Twaalfhoven H et al (2016) Validation of soluble amyloid-beta precursor protein assays as diagnostic CSF biomarkers for neurodegenerative diseases. J Neurochem 137(1):112-121. https://doi.org/10.1111/jnc.13527

22. Camby I, Le Mercier M, Lefranc F, Kiss R (2006) Galectin-1: a small protein with major functions. Glycobiology 16(11):137R157R. https://doi.org/10.1093/glycob/cwl025

23. Horie H, Kadoya T (2002) Galectin-1 plays essential roles in adult mammalian nervous tissues. Roles of oxidized galectin-1. Glycoconj J 19(7):479-489. https://doi.org/10.1023/b:glyc. 0000014077.84016 .52

24. Stancic M, van Horssen J, Thijssen VL, Gabius HJ, van der Valk P, Hoekstra D, Baron W (2011) Increased expression of distinct galectins in multiple sclerosis lesions. Neuropathol Appl Neurobiol 37(6):654-671. https://doi.org/10.1111/j.1365-2990. 2011.01184.x

25. Sakaguchi M, Imaizumi Y, Okano H (2007) Expression and function of galectin-1 in adult neural stem cells. Cell Mol Life Sci 64(10):1254-1258. https://doi.org/10.1007/s00018-007-6476-5

26. Chen HL, Liao F, Lin TN, Liu FT (2014) Galectins and neuroinflammation. Adv Neurobiol 9:517-542. https://doi.org/10.1007/ 978-1-4939-1154-7_24

27. McGraw J, Gaudet AD, Oschipok LW, Kadoya T, Horie H, Steeves JD, Tetzlaff W, Ramer MS (2005) Regulation of neuronal and glial galectin-1 expression by peripheral and central axotomy of rat primary afferent neurons. Exp Neurol 195(1):103-114. https://doi.org/ 10.1016/j.expneurol.2005.04.004

28. McGraw J, McPhail LT, Oschipok LW, Horie H, Poirier F, Steeves JD, Ramer MS, Tetzlaff W (2004) Galectin-1 in regenerating motoneurons. Eur J Neurosci 20(11):2872-2880. https://doi.org/10. 1111/j.1460-9568.2004.03802.x

29. Chang-Hong R, Wada M, Koyama S, Kimura H, Arawaka S, Kawanami T, Kurita K, Kadoya T et al (2005) Neuroprotective effect of oxidized galectin-1 in a transgenic mouse model of amyotrophic lateral sclerosis. Exp Neurol 194(1):203-211. https://doi. org/10.1016/j.expneurol.2005.02.011

30. Zhang L, Chang M, Li H, Hou S, Zhang Y, Hu Y, Han W, Hu L (2007) Proteomic changes of PC12 cells treated with proteasomal inhibitor PSI. Brain Res 1153:196-203. https://doi.org/10.1016/j. brainres.2007.03.073

31. Werner CJ, Heyny-von Haussen R, Mall G, Wolf S (2008) Proteome analysis of human substantia nigra in Parkinson's disease. Proteome Sci 6:8. https://doi.org/10.1186/1477-5956-6-8

32. Marques TM, Van Rumund A, Kuiperij HB, Verbeek MM (2017) Biomarkers in cerebrospinal fluid for synucleinopathies, tauopathies, and other neurodegenerative disorders. Handbook Clin Neurol 146:99-113. https://doi.org/10.1016/b978-0-12804279-3.00007-1

33. Quintá HR, Pasquini JM, Rabinovich GA, Pasquini LA (2014) Glycan-dependent binding of galectin-1 to neuropilin-1 promotes axonal regeneration after spinal cord injury. Cell Death Differ 21(6):941-955. https://doi.org/10.1038/cdd.2014.14

34. Wang H, Wu M, Zhan C, Ma E, Yang M, Yang X, Li Y (2012) Neurofilament proteins in axonal regeneration and neurodegenerative diseases. Neural Regen Res 7(8):620-626. https://doi.org/10. 3969/j.issn. 1673-5374.2012.08.010

35. Teunissen CE, Petzold A, Bennett JL, Berven FS, Brundin L, Comabella M, Franciotta D, Frederiksen JL et al (2009) A consensus protocol for the standardization of cerebrospinal fluid collection and biobanking. Neurology 73(22):1914-1922. https://doi.org/10. 1212/WNL.0b013e3181c47cc2

36. Willemse EAJ, van Uffelen KWJ, van der Flier WM, Teunissen CE (2017) Effect of long-term storage in biobanks on cerebrospinal fluid biomarker $A \beta(1-42)$, T-tau, and P-tau values. Alzheimers Dement Diagn Assess Dis Monit 8:45-50. https://doi.org/10. 1016/j.dadm.2017.03.005 